\title{
Pentraxin 3, a novel inflammatory marker in heart failure patients: its expression in whole blood as a function of clinical severity
}

\author{
Cabiati $\mathrm{M}^{1 *}$, Salvadori $\mathrm{C}^{2}$, Verde $\mathrm{A}^{3}$ and Del Ry $\mathrm{S}^{1}$ \\ ${ }^{1}$ Laboratory of Biochemistry and Molecular Biology, Institute of Clinical Physiology CNR, Pisa, Italy \\ ${ }^{2}$ Laboratory of Medical Science, Institute of Life Sciences, Scuola Superiore Sant'Anna, Pisa, Italy \\ ${ }^{3}$ Department of Cardiac Surgery, Niguarda Ca' Granda Hospital, Milan / Piacenza Civil Hospital, Italy
}

\begin{abstract}
Recent studies have shown that plasma long pentraxin PTX3 levels were increased in heart failure (HF) patients with advancing NYHA functional class, but the modulation of PTX3 mRNA expression in these different classes is not yet settled. Aim of the study was to evaluate PTX3 expression in whole blood of HF patients according to clinical severity and in a group of age-matched healthy subjects, as control. In order to realize a multi-axis framework, Nt-proBNP and CRP plasma levels were also measured. Total RNA was extracted from whole blood of HF patients (NYHA II n=10; NYHA III- $I V$ n=13) and 8 C with PAXgene Blood RNA Kit, and PTX3 mRNA was evaluated by Real-Time PCR. Plasma NT-proBNP and CRP were measured by a fully automated immunoassay. PTX3 expression resulted significantly higher in $\mathrm{HF}$ patients at different disease stages with respect to $\mathrm{C}(\mathrm{C}=2.2 \pm 0.8, N Y H A I I=3.0 \pm 1.3, N Y H A I I I-I V=9.7 \pm 2.8 ; \mathrm{p}=0.02 \mathrm{C}$ vs $N Y H A I I, \mathrm{p}=0.03$ $N Y H A I I$ vs NYHA III- $I V$ ). At plasma levels of HF patients, no changes were found for CRP while NT-proBNP was significantly higher in function of clinical severity. The high PTX3 mRNA levels found in HF patients, supporting its role as an acute-phase response protein during HF, able to predict their inflammatory status.
\end{abstract}

\section{Introduction}

The clinical syndrome of heart failure (HF) is characterized by a systemic inflammatory response that contributes to end organ damage in the heart and circulation, leading to worsening symptoms. The inflammation process has a pivotal role in the clinical syndrome of heart failure (HF) leading to cardiac remodelling; in fact, $\mathrm{HF}$ is characterized by the secretion and production of a portfolio of pro-inflammatory cytokines and inflammatory mediators that appear to contribute to disease progression [1]. Systemic biomarkers such as C-reactive protein (CRP), interleukin (IL)- 6 , tumor necrosis factor (TNF)- $\alpha$, flogistic molecules CD40 and its soluble ligand, adhesion molecules ICAM-1, VCAM-1, E-selectin and P-selectin as well as NT-proBNP, troponin $\mathrm{T}$, and fibrinogen, are useful indices for evaluating inflammation and tissue damage associated with HF [2-4]. In addition to these mediators, a more important vascular inflammatory marker, the prototypic long pentraxin3, PTX3, is a fundamental player in immunity and inflammation [5]. The pentraxin superfamily is characterized by the presence of the "pentraxin domain", composed of a conserved 8-aminoacid long sequence, in the carboxy-terminal region [5]. This family includes the short and long pentraxins, which differ in the primary structure of the protein; CRP and serum amyloid P-component (SAP) are two well-characterized-short pentraxins of about $25-\mathrm{kDa}$, mainly produced in the liver in response to proinflammatory mediators such as IL-6 [5,6], while the long pentraxin, PTX3, consists of a major $45-\mathrm{kDa}$ form of monomers, which are assembled to form multimers predominantly of $440 \mathrm{kDa}$ molecular mass [5]. PTX3 is rapidly produced and released by several cell types, including leukocytes, dendritic cells, fibroblasts, adipocytes and endothelial cells, in response to local inflammation in the cardiovascular system [5-8]. In recent years, the presence of PTX3 was detected in the myocardium and in the vasculature under different pathological conditions, which was paralleled by the observation of increased plasma PTX3 levels in patients with cardiovascular disorders [9]. These results drove research toward the investigation of a PTX3 role as earlier biomarker, mainly in cardiovascular disease. Recent studies have shown that in HF, plasma PTX3 levels correlate with advancing severity [10-13] while it was very low in normal conditions, but the modulation of PTX3 mRNA expression in different NYHA (New York Heart Association) classes is not yet settled.

The study aimed to evaluate the presence of PTX3 expression profile in whole blood of HF patients according to clinical severity and in a group of age-matched healthy subjects as control, applying RealTime PCR technique in a small amount of whole blood.

\section{Materials and methods}

Study design: patient's enrolment, inclusion and exclusion criteria

This study complied with the principles of the Declaration of Helsinki, and the protocol was approved by the Niguarda Hospital Ethics Committee $\left(\mathrm{n}^{\circ} 176 / 05\right)$. Informed consent was obtained from

*Correspondence to: Cabiati Manuela, CNR Institute of Clinical Physiology, Via Giuseppe Moruzzi 1, 56124 Pisa, Italy, Tel: +39 050 3153551, Fax +39050 3152166, E-mail: manuela.cabiati@ifc.cnr.it

Key words: PTX3, Heart failure, NYHA class, Leukocytes, mRNA expression

Received: September 05, 2018; Accepted: September 17, 2018; Published: September 20, 2018 
all individual participants included in the study. The study was carried out on twenty-three patients with HF (New York Heart Classification patients NYHA II, $\mathrm{n}=10$ and NYHA III-IV $\mathrm{n}=13$ ) and reduced left ventricular ejection fraction (LVEF) that were potentially eligible for heart transplant.

These patients had been admitted for routine re-evaluation (echocardiography, cardiopulmonary exercise test and right heart catheterisation) or for acute decompensation of chronic HF and all of them had an optimal medical therapy plus cardiac resynchronisation therapy when indicated by guidelines criteria. Inclusion criteria consisted of a significantly depressed LVEF $(<45 \%)$, measured by echocardiography. Exclusion criteria were acute myocardial infarction or unstable angina within 6 months before the examination, significant primitive pulmonary disease, and renal failure (defined as a serum creatinine value above $2.0 \mathrm{mg} / \mathrm{dl}$ ). Patients were treated with restriction of water-sodium intake (using a personalized, well-controlled diet with a sodium intake of 100-140 $\mathrm{mmol} /$ day) and were receiving homogeneous optimal medical treatment (furosemide, ACE-inhibitors, carvedilol, spironolactone for patients in class NYHA $\geq \mathrm{III}$ ), not discontinued at the time of the study, for ethical reasons.

This study is a part of a larger project in which the patients were deeply characterized and, as reported in previous studies of ours [1416], plasma IL-6, IL-8 and TNF-a levels resulted increased in patients with worsening symptoms. Eight healthy and age-matched subjects with normal LV function (LVEF $>55 \%$ ) and no history of cardiovascular events were enrolled as control group.

\section{Blood collection, reverse transcription and real time PCR}

The peripheral blood samples $(2.5 \mathrm{ml})$ were withdrawal from both HF patients and control group and collected in PAXgene Blood RNA tubes (DIALAB ITALIA Srl), an innovative methodology for the collection, storage and transport of blood, which efficiently stabilizes intracellular RNA and allows preservation of the samples at -20 to $-70^{\circ} \mathrm{C}$, maintaining the same degree of purity and stability of the fresh blood. RNA was isolated with a PAXgene Blood RNA kit (Qiagen, Milan, Italy) to obtain total RNA, as previously described $[14,15,17$ 19]. Briefly, blood samples were processed manually and purification began with a centrifugation step. The pellet was washed, resuspended and incubated in a specific buffer together with proteinase $\mathrm{K}$ for protein digestion. RNA was selectively bound to the PAXgene silica membrane while contaminants were removed and finally eluted in an elution buffer and heat-denatured. The samples were stored at $-80^{\circ} \mathrm{C}$, evaluated spectrophotometrically, and reversely transcribed with a iScript cDNA Synthesis Kit (Bio-Rad) according to the manufacturer's instructions.

Reference gene primer pairs were designed with Primer Express Version 2.0 (Applied Biosystems) as reported in a previous work of ours $[14,15,17-19]$ as well as PTX3 primer sequence (GenBank $n^{\circ}$ : NM_002852; forward:AATGCTGTGTCTCTGTCA; reverse:ACAT ACCAATAACAATGAACAATG). Real-Time PCR reactions were performed in a 96-well CFX96 RT-PCR System (Bio-Rad Laboratories Inc., Hercules, CA, USA) in duplicate and carried out in a total volume of $25 \mu \mathrm{l}$ per reaction including $2 \mu \mathrm{l}$ of template cDNA, $1 \mu \mathrm{M}$ of each primer, 2X QuantiFast SYBR Green SuperMix (Qiagen) and sterile water. Reaction conditions of all primer pairs used were optimized. The MIQE guideline were strictly followed [20].

\section{NT-proBNP and C-reactive protein assays}

To provide complementary information about HF state, the $\mathrm{N}$-terminal fragment of brain natriuretic peptide (NT-proBNP) and CRP plasma levels were also measured and compared with PTX3 mRNA expression. Each sample was rapidly separated and stored at $-20^{\circ} \mathrm{C}$ until assay. NT-proBNP was measured by a fully automated electrochemiluminescence analyzer, while CRP concentrations using a Roche/Hitachi 917 Analyzer by high-sensitive immuno-nephelometric method (both of Roche Diagnostics GmbH, Mannheim, Germany).

\section{Statistical analysis}

Real-time PCR data were analyzed using the $2^{-\Delta \Delta \mathrm{Ct}}$ method obtained using Bio-Rad's CFX96 manager software and expression levels of PTX3 was normalized with the geometric mean of the three most stably expressed genes (the tyrosine 3-monooxygenase/tryptophan 5-monooxygenase activation protein $\mathrm{z}$ polypeptide, YWHAZ; the topoisomerase II Beta, TOP $2 b$; the signal recognition particle $14 \mathrm{kDa}$., SRP14) established in a previous work $[17,19]$.

Fisher's test after ANOVA was applied to assess differences between more than two independent groups, whereas unpaired Student's $t$-test was employed for differences between two independent groups; all data were analyzed by using Statview 5.0.1 (SAS Institute, Inc., Cary, NC, USA). The results are expressed as mean \pm SEM and $p$-value was considered significant when $<0.05$. Since biomarkers values were not normally distributed, both the original and the logarithmic transformation of data were used for statistical analysis. The association between different variables was tested by Pearson correlation test.

\section{Results}

\section{Clinical findings in HF patients}

The clinical and biochemical characteristics of each HF patient, subdivided following their class of worsening symptoms, are described in Table 1. Median age of failing patients was comparable between the NYHA classes as was left ventricular impairment, evaluated by echocardiographic measurements.

\section{Assessment of Real-time PCR reaction in whole blood}

Real-time PCR optimal reaction conditions for human PTX3 were empirically determined: $\mathrm{T} a$ of $62^{\circ} \mathrm{C}$ and 42 cycles. Dilution series were run for PTX3 to quantify the efficiency that resulted $105 \%$ and a linear standard curve, $\mathrm{R}^{2}$, of 0.998 .

\section{PTX3 gene expression profiling in whole blood of HF patients and controls}

Failing patients had higher PTX3 mRNA expression than control (Figure 1). PTX3 transcriptional profiling were significantly elevated in HF patients with highest NYHA class (C vs NYHA III-IV, p=0.02). A significant increase was also observed between NYHA II and NYHA III-IV ( $\mathrm{p}=0.03)$. No statistically significant correlations were found between PTX3 with echocardiography parameters.

\section{Plasma biomarkers evaluation: neuro-humoral and inflammatory states in HF patients}

In HF patients, NT-proBNP plasma levels were significantly higher in function of clinical severity (NYHA $I I=717 \pm 41 \mathrm{pg} / \mathrm{ml}$, NYHA III$I V=2764 \pm 572 \mathrm{pg} / \mathrm{ml} ; \mathrm{p}=0.03)$ as well as with respect to reference values of control $(0-155 \mathrm{pg} / \mathrm{ml})$. Conversely, no significant difference was found for the circulating flogosis indices, as CRP, in these different classes (Figure 2). No significant correlations were observed between the biomarkers analysed and PTX3.

\section{Discussion}

Advances in bio-molecular technologies have led to the discovery of many novel biomarkers providing important information, which can 
Table 1. Clinical characteristics of heart failure patients according to NYHA class

\begin{tabular}{|c|c|c|c|}
\hline & $\begin{array}{l}\text { Patients } \\
\text { NYHA II } \\
(\mathrm{n}=10)\end{array}$ & $\begin{array}{c}\text { Patients } \\
\text { NYHA III-IV } \\
\quad(n=13)\end{array}$ & $p$ \\
\hline Age, yrs & $54 \pm 4$ & $53 \pm 2$ & ns \\
\hline Male, n (\%) & $10(100)$ & $11(78)$ & ns \\
\hline BMI, $\mathrm{Kg} / \mathrm{m}^{2}$ & $26 \pm 2$ & $26 \pm 1$ & $\mathrm{~ns}$ \\
\hline Hypertension, n (\%) & $1(10)$ & $1(7)$ & $\mathrm{ns}$ \\
\hline Diabetes, $\mathrm{n}(\%)$ & $1(10)$ & $3(20)$ & ns \\
\hline Dislypidemia, n (\%) & $2(10)$ & $5(36)$ & ns \\
\hline Smoke, $\mathrm{n}(\%)$ & $4(40)$ & $3(20)$ & ns \\
\hline CAD familiarity, n (\%) & $3(37)$ & $4(44)$ & ns \\
\hline Etiology, n (\%) & & & ns \\
\hline IDC & $9(90)$ & $10(77)$ & \\
\hline IHD & $1(10)$ & $3(29)$ & \\
\hline LVEF, \% & $31 \pm 4$ & $26 \pm 2$ & $\mathrm{~ns}$ \\
\hline LVEDV, ml & $189 \pm 31$ & $281 \pm 32$ & 0.043 \\
\hline LVESV, ml & $137 \pm 27$ & $211 \pm 27$ & 0.05 \\
\hline LVEDD, mm & $61 \pm 4$ & $74 \pm 3.8$ & $\mathrm{~ns}$ \\
\hline LVPPT, mm & $9.6 \pm 0.2$ & $8.2 \pm 0.75$ & ns \\
\hline IST, mm & $9.0 \pm 0.7$ & $8.25 \pm 0.43$ & ns \\
\hline MID, n (\%) & & & 0.0105 \\
\hline 0 & $1(10)$ & - & \\
\hline $1+$ & $5(50)$ & $2(8)$ & \\
\hline $2+$ & $3(30)$ & $7(54)$ & \\
\hline $3+$ & $1(10)$ & $4(31)$ & \\
\hline $4+$ & - & - & \\
\hline LA area, $\mathrm{cm}^{2}$ & $21 \pm 2$ & $40.3 \pm 7.5$ & 0.04 \\
\hline TAPSE, mm & $17 \pm 1$ & $15.1 \pm 1.2$ & ns \\
\hline \multicolumn{4}{|l|}{ Therapy, n (\%) } \\
\hline ACEi & $7(70)$ & $7(54)$ & ns \\
\hline$\beta$-Blocker & $10(100)$ & $13(100)$ & ns \\
\hline Statin & $5(50)$ & $6(46)$ & ns \\
\hline Antiplatelet & $7(70)$ & $7(54)$ & ns \\
\hline Diuretic & $8(80)$ & $13(100)$ & ns \\
\hline Creatinine, $\mathrm{mg} / \mathrm{dL}$ & $1.12 \pm 0.18$ & $1.12 \pm 0.08$ & ns \\
\hline Urea, mg/dL & $46.7 \pm 9.8$ & $54.6 \pm 6.5$ & ns \\
\hline Total bilirubine, $\mathrm{mg} / \mathrm{dL}$ & $0.76 \pm 0.14$ & $1.30 \pm 0.27$ & ns \\
\hline
\end{tabular}

Data are expressed as mean and SE or frequency (percentage)

ACEi: Angiotensin converting enzyme inhibitor; BMI: Body mass index; CAD: Coronary artery disease; IDC: Idiopathic dilated cardiomyopathy; IHD: Ischemic heart disease; IST: Interventricular septum thickness; LA: Left atrium; LVEDD: Left ventricular end-diastolic diameter; LVEDV: Left ventricular end-diastolic volume; LVEF: Left ventricular ejection fraction; LVESV: Left ventricular end-systolic volume; LVPPT: Left ventricular posterior wall thickness; MID: Mitral insufficiency grade; NYHA: New York Heart Classification; TAPSE: Tricuspid annular plane systolic excursion

be used in disease screening and diagnosis, determining prognoses, and therapeutic monitoring of patients. In this study we evaluated in HF patients an important inflammatory biomarker, the PTX3, measuring its mRNA expression levels directly in circulating blood cells. This approach was integrated with the evaluation of two classic neurohormonal and inflammatory markers as NT-proBNP and CRP, but at the plasma level, since they are not detectable on leukocytes.

The most interesting result of the present study was the possibility of measuring the PTX3 expression which showed significantly elevated mRNA expression in HF patients as a function of clinical severity. Our findings are in line with several studies where plasma PTX3 levels were found to be increased in HF patients associated with a higher risk to develop cardiac events [12,13,21-23].

The transcriptional trend of PTX3 was found to be significantly elevated in NYHA III-IV with respect to NYHA II, as also observed by Duran S. et colleagues [24], which revealed an increase of this early marker in plasma of failing patients, suggesting a pivotal role for PTX3 in the pathogenesis of cardiac failure.

It is well-known that the clinical syndrome of HF is characterized by a systemic inflammatory response that contributes to end-organ damage in the heart and circulation leading to worsening symptoms [25]. PTX3 is a key candidate for local early inflammatory response able to predict the inflammatory status of HF patients. Therefore, since long PTX3 is mainly produced by peripheral blood leucocytes, macrophages and neutrofils and by endothelium, its levels may better reflect the inflammatory status of the vascular bed; in addition, circulating PTX3 may also derive from injured myocardium, because this marker is also expressed by cardiomyocytes, strengthening the evidence for the role of this protein in cardiac remodeling $[7,11]$. When we compared our data we found a statistical difference in PTX3 mRNA expression between patients of NYHA II with respect to those in III-IV class. These results having been obtained at mRNA expression level appear to sustain findings of Latini et al. [26], which reported that increased PTX3 plasma concentration is associated with worse outcome in HF patients. In the light of our previous studies, where in the same set of patients was characterized the mRNA profile of C-type natriuretic peptide [14,18], adrenomedullin and intermedin [15] as well as the modulation of adenosine receptors $[19,20]$ in the same set of patients

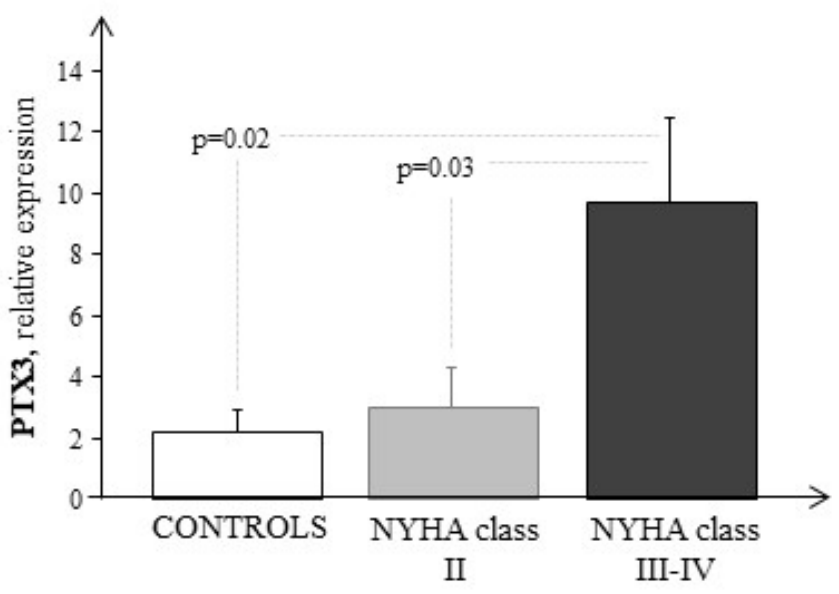

Figure 1. Comparison between PTX3 transcriptional profiling in whole blood of HF patients as a function of clinical severity (grey and black bars) and of healthy subjects (white bar). Data are expressed as mean \pm SEM

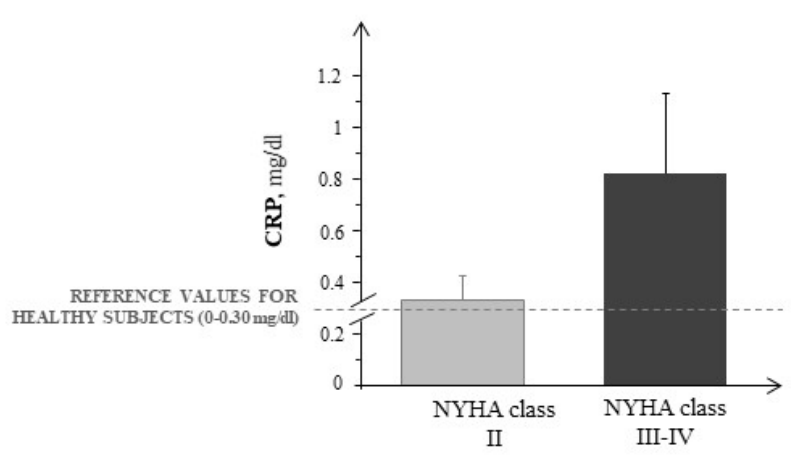

Figure 2. CRP plasma levels in the different NYHA classes of HF patients (grey and black bars). Data are expressed as mean \pm SEM 
was characterized, the ability to measure also an important index of systemic inflammation directly in whole blood, especially leukocytes, avoiding more invasive investigation was of pivotal importance. Furthermore, the technique used in this study allowed us to evaluate the PTX3 expression profile by exploiting a rapid and easy procedure for the preparation of nucleic acid starting from small quantity of whole blood and avoiding a more invasive survey for HF patients. In addition, the PAXgene blood RNA extraction have permitted to obtain suitable quantity of total RNA, from which analyze simultaneously different biomarkers involved in different processes underlying HF, as inflammation. In order to provide a solid basis for the mRNA expression study and to better appreciate the pathophysiology of HF, in this study was examined the combination of plasma NT-proBNP, a consolidated cardiac marker, and CRP with circulating PTX3. In fact, this sort of "multi-axis framework" provides complementary information and aids in both stratifying risk more effectively among patients with cardiovascular diseases and addressing the best therapy $[22,26]$. Although the association of both NT-proBNP and CRP with prognosis in HF is well-established, this approach could help us to understand cardiac changes that can occur in HF patients. In this study both biomarkers increased in HF patients in advanced NYHA class, but a close and precise correlation with PTX3 is lacking, probably due to the small number of patients analyzed. However, it has been reported that elevated high-sensitive CRP has an independent prognostic value in HF patients [26-29], but PTX3 provides important diagnostic and prognostic information and is a more sensitive and specific marker than CRP in these patients, showing also a better predictive value than CRP. The possibility of analysing different biomarkers both at plasma and mRNA levels simultaneously, such as neurohormonal markers that reveal pressure and/or volume overload, markers of myocardial damage and markers of inflammation, could be a reliable method for stratifying patients with $\mathrm{HF}$.

In conclusion, the findings of this paper demonstrating increased PTX3 at transcriptional levels reinforced data about its increase in plasma of HF patients, supporting a role for PTX3 as an important biomarker able to predict the inflammatory status of HF patients, as an acute-phase response protein. Although clinical and experimental studies will be necessary, the evidence for both a regulatory and predictive role in the pathogenesis of cardiac diseases provides further stimuli for assessing the clinical relevance of PTX3 measurement in cardiovascular disorders.

\section{Limitations}

The major limitation of this study are represented by the small number of patients enrolled, due to the fact that recruitment of patients meeting the inclusion criteria was limited to a single Center, and the lack of a concomitant evaluation of PTX3 plasma concentration. This study being part of a larger project, as stated in material and methods, where many biomarkers were investigated both by immunometric assay than by Real-Time PCR analysis and, while for bio-molecular study is necessary a small quantity of cDNA, the immunometric assay needs of higher quantity of plasma making the samples unavailable to further use. These results, together with our previously published data $[14,15,18-20]$ open the door to the possibility to evaluate a panel of multiple markers simultaneously from a single biological sample maximizing the impact of technological advances on clinical care.

\section{Fundings}

This work was supported by Institutional Grants of National Research Council (CNR).

\section{Conflicts of interest}

The authors declare that there is no conflict of interest regarding the publication of this article.

\section{Author contributions}

MC, SDR conceptualized and designed the study, drafted the manuscript, and reviewed and revised the manuscript.

MC, CS, SDR designed the data collection instruments, collected data, carried out the initial analyses, and reviewed and revised the manuscript.

\section{$\mathrm{AV}$ enrolled patients and revised the manuscript.}

All authors approved the final manuscript as submitted and agree to be accountable for all aspects of the work.

The paper is in loving memory of our friend Dr Raffaele Caruso, prematurely deceased during the writing of this manuscript.

\section{References}

1. Anker SD, von Haehling S (2004) Inflammatory mediators in chronic heart failure: an overview. Heart 90: 464-470. [Crossref]

2. Vasan RS, Sullivan LM, Roubenoff R (2003) Inflammatory markers and risk of heart failure in elderly subjects without prior myocardial infarction: The framingham heart study. Circulation 107: 1486-1491. [Crossref]

3. Anand IS, Latini R, Florea VG (2005) C-reactive protein in heart failure: prognostic value and the effect of valsartan. Circulation 112: 1428-1434. [Crossref]

4. Braunwald E (2008) Biomarkers in heart failure. N Engl J Med 358: 2148-2159. [Crossref]

5. Garlanda C, Bottazzi B, Bastone A (2005) Pentraxins at the crossroads between innate immunity, inflammation, matrix deposition, and female fertility. Ann Rev Immunol 23: 337-366.

6. Pepys MB, Hirschfield GM (2003) C-reactive protein: a critical update. J Clin Inves 111: 1805-1812. [Crossref]

7. Breviario F, d'Aniello EM, Golay J (1992) Interleukin-1-inducible genes in endothelial cells. Cloning of a new gene related to C-reactive protein and serum amyloid $\mathrm{P}$ component. J Biol Chem 267: 22190-22197.

8. Bonacina F, Baragetti A, Catapano AL (2013) Long pentraxin 3: experimental and clinical relevance in cardiovascular diseases. Mediators Inflamm 2013: 725102.

9. Norata GD, Garlanda C, Catapano AL (2010) The long pentraxin PTX3: a modulator of the immunoinflammatory response in atherosclerosis and cardiovascular diseases. Trends Cardiovasc Med 20: 35-40.

10. Kaess BM, Vasan RS (2011) Heart failure: pentraxin 3â€’a marker of diastolic dysfunction and HF? Nat Rev Cardiol 8: 246-248. [Crossref]

11. Nebuloni M, Pasqualini F, Zerbi P (2011) PTX3 expression in the heart tissues of patients with myocardial infarction and infectious myocarditis. Cardiovasc Pathol 2: 27-35.

12. Kotooka N, Inoue T, Aoki S, Anan M, Komoda H, et al. (2008) Prognostic value of pentraxin 3 in patients with chronic heart failure. Int J Cardiol 130: 19-22. [Crossref]

13. Suzuki S, Takeishi Y, Niizeki T (2008) Pentraxin 3, a new marker for vascular inflammation, predicts adverse clinical outcomes in patients with heart failure. $\mathrm{Am}$ Heart $J$ 155: 75-81.

14. Cabiati M, Sabatino L, Caruso R (2013) C-type natriuretic peptide transcriptomic profiling increases in human leukocytes of patients with chronic heart failure as a function of clinical severity. Peptides 47: 110-114.

15. Cabiati M, Sabatino L, Svezia B, Caruso R, Verde A, et al. (2014) Adrenomedullin and intermedin gene transcription is increased in leukocytes of patients with chronic heart failure at different stages of the disease. Peptides 55: 13-16. [Crossref]

16. Schiller NB, Shah PM, Crawford M (1989) Recommendations for quantitation of the left ventricle by two-dimensional echocardiography. American society of echocardiography committee on standards, subcommittee on quantitation of twodimensional echocardiograms. J Am Soc Echocardiogr 2: 358-367. [Crossref]

17. Cabiati M, Sabatino L, Caruso R (2012) Gene expression of C-type natriuretic peptide and of its specific receptor NPR-B in human leukocytes of healthy and heart failure subjects. Peptides 37: 240-246. [Crossref] 
18. Cabiati M, Caruso R, Verde A (2013) Transcriptomic profiling of the four adenosine receptors in human leukocytes of heart failure patients. Biomed Res Int 2013: 569438.

19. Sabatino L, Cabiati M, Caselli C (2013) Adenosine receptor expression and gene reference evaluation in human leukocytes. Clin Lab 59: 571-577.

20. Bustin SA, Benes V, Garson JA, Hellemans J, Huggett J, et al. (2009) The MIQE guidelines: minimum information for publication of quantitative real-time PCR experiments. Clin Chem 55: 611-622. [Crossref]

21. Caruso R, Verde A, Cabiati M (2012) Association of pre-operative interleukin-6 levels with Interagency Registry for Mechanically Assisted Circulatory Support profiles and intensive care unit stay in left ventricular assist device patients. J Heart Lung Transplant 31: 625-633.

22. Ishino M, Takeishi Y, Niizeki T (2008) Risk stratification of chronic heart failure patients by multiple biomarkers: implications of BNP, H-FABP, and PTX3. Circ J 72: 1800-1805. [Crossref]

23. Latini R, Maggioni AP, Peri G (2004) Lipid assessment trial italian network (latin) investigators. prognostic signi?cance of the long pentraxin ptx 3 in acute myocardial infarction. Circulation 110: 2349-54.
24. Duran S, Duran I, Kaptanagasi FAO, Nartop F, Ciftci H, et al. (2013) The role of pentraxin 3 as diagnostic value in classification of patients with heart failure. Clin Biochem 46: 983-987. [Crossref]

25. Hartupee J, Mann DL (2013) Positioning of inflammatory biomarkers in the heart failure landscape. J Cardiovasc Transl Res 6: 485-492. [Crossref]

26. Latini R, Gullestad L, Masson S, Nymo SH, Ueland T (2012) Pentraxin-3 in chronic heart failure: the CORONA and GISSI-HF trials. Eur J Heart Fail 14: 992-999. [Crossref]

27. Rector TS, Anand IS (2010) Research needed to support clinical use of biomarkers as prognostic indicators for patients with heart failure. Cardiol Res Pract 2010: 453851.

28. Mueller C, Laule-Kilian K, Christ A, Brunner-La Rocca HP, Perruchoud AP (2006) Inflammation and long-term mortality in acute congestive heart failure. Am Heart $J$ 151: 845-850. [Crossref]

29. Yin WH, Chen JW, Jen HL, Chiang MC, Huang WP, et al. (2004) Independent prognostic value of elevated high-sensitivity C-reactive protein in chronic heart failure. Am Heart J 147: 931-938. [Crossref]

Copyright: (C)2018 Cabiati M. This is an open-access article distributed under the terms of the Creative Commons Attribution License, which permits unrestricted use, distribution, and reproduction in any medium, provided the original author and source are credited. 